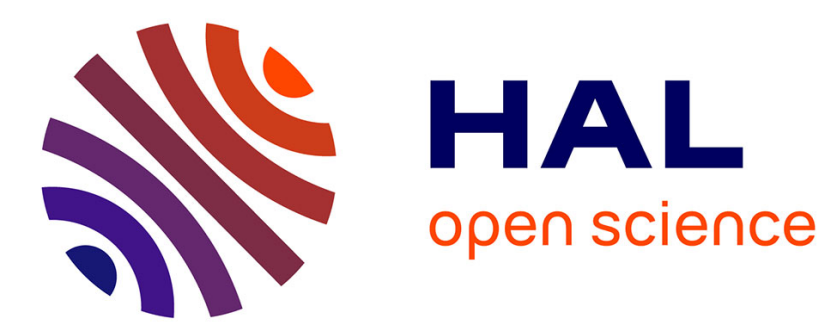

\title{
Self-calibrating nonlinear reconstruction algorithms for variable density sampling and parallel reception MRI
}

\author{
Loubna El Gueddari, C. Lazarus, H Carrié, A. Vignaud, Philippe Ciuciu
}

\section{To cite this version:}

Loubna El Gueddari, C. Lazarus, H Carrié, A. Vignaud, Philippe Ciuciu. Self-calibrating nonlinear reconstruction algorithms for variable density sampling and parallel reception MRI. 10th IEEE Sensor Array and Multichannel Signal Processing workshop, Jul 2018, Sheffield, United Kingdom. pp.1-5. hal-01782428v2

\author{
HAL Id: hal-01782428 \\ https://hal.inria.fr/hal-01782428v2
}

Submitted on 20 Jun 2018

HAL is a multi-disciplinary open access archive for the deposit and dissemination of scientific research documents, whether they are published or not. The documents may come from teaching and research institutions in France or abroad, or from public or private research centers.
L'archive ouverte pluridisciplinaire HAL, est destinée au dépôt et à la diffusion de documents scientifiques de niveau recherche, publiés ou non, émanant des établissements d'enseignement et de recherche français ou étrangers, des laboratoires publics ou privés. 


\title{
SELF-CALIBRATING NONLINEAR RECONSTRUCTION ALGORITHMS FOR VARIABLE DENSITY SAMPLING AND PARALLEL RECEPTION MRI
}

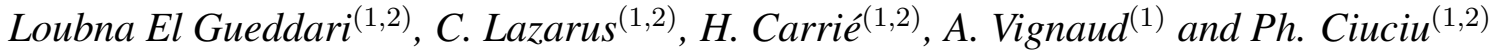 \\ ${ }^{(1)}$ CEA/NeuroSpin, Bât 145, F-91191 Gif-sur Yvette, France. \\ ${ }^{(2)}$ INRIA-CEA Saclay Ile-de-France, Parietal team, Saclay, France.
}

\begin{abstract}
Compressed Sensing has allowed a significant reduction of acquisition times in MRI, especially in the high resolution (e.g., $400 \mu \mathrm{m}$ ) context. However, in this setting CS must be combined with parallel reception as multichannel coil acquisitions maintain high input signal-to-noise ratio (SNR). To get rid of usual parallel imaging limitations (output SNR loss), non-Cartesian trajectories provide a gain in sampling efficiency in the CS context. In this paper, we propose a self-calibrating MRI reconstruction framework that handles variable density sampling. Low resolution sensitivity maps are estimated from the low frequency $k$-space content using an original and fast method while MR images are reconstructed using a nonlinear iterative algorithm, which promotes sparsity in the wavelet domain. As regards the optimization task, we compare three firstorder proximal gradient methods: Forward Backward (FB), Fast Iterative Soft Thresolding Algorithm (FISTA) and Proximal Optimized Gradient Method (POGM) and evaluate their respective convergence speed. Comparison with state-of-the-art (i.e., $\ell_{1}$-ESPIRiT) suggests that our self-calibrating POGM-based algorithm outperforms current approaches both in terms of image quality and computing time on several acquired data collected at 7 Tesla and we will focus more specifically on prospective non-Cartesian 8-fold accelerated in vivo Human brain data.
\end{abstract}

Index Terms - Prospective compressed sensing, parallel reception, non-Cartesian trajectories, nonlinear reconstruction.

\section{INTRODUCTION}

Magnetic Resonnance Imaging (MRI) is a key imaging technique to probe soft tissues (e.g., the brain) non-invasively. However, its acquisition time may be prohibitive in the context of high resolution imaging. To cope with this issue, parallel imaging (PI) has been introduced in the late 90 s to accelerate the acquisition by undersampling the $k$-space along the phase encoding direction [1], while collecting these data over a phased array coil, whose multiple receivers are spatially specific. This makes MR image reconstruction feasible by unfolding subsampling artifacts in the image domain (Sensitivity Encoding approaches) [1] or by interpolating $k$ space for filling missing data (GRAPPA-based approaches) [2]. For a recent overview, see [3].

More recently, Compressed Sensing (CS) theory [4, 5] has been successfully applied to MRI for decreasing even more acquisition times while providing theoritical guarantees of exact reconstruction in some particular cases (e.g., noise-free) [6]. This theory is based

The authors wish to acknowledge the COSMIC project funded by the CEA DRF-Impulsion call in 2016. They are also grateful to Prof. Jeff Fessler who provides significant help during his visit to NeuroSpin in June 2017. on three main pillars: (i) sparsity or compressibility of MR images in a given dictionary (e.g., wavelets, curvelets, ...), (ii) incoherence between sensing and sparsity bases leading to pseudo-random sampling schemes and (iii) nonlinear image reconstruction for promoting image sparsity in the wavelet domain. Since then, the design of incoherent but physically plausible sampling schemes on the one hand, and the implementation of efficient reconstruction algorithms on the other hand have become active research topics. A first key point lies in the implementation of variable density sampling to minimize the number of measurements. This was first empirically evidenced in [6] and then theoretically proved [7-9]. A second important concern for prospective CS is the feasability of the corresponding $k$-space trajectory on a real scanner: it has to comply with hardware constraints such as its maximal gradient magnitude and slew rate. To optimize its sampling efficiency, the $k$-space trajectory (e.g., radial spokes [10], spiral [11], ...) no longer lies on a Cartesian grid, hence optimal image reconstruction algorithms make use of the fast nonequispaced Fast Fourier transform (NFFT) [12,13], which takes a non-uniformally sampled $k$-space as input data and returns an image on the Cartesian grid.

For MR image reconstruction, many nonlinear iterative algorithms have been proposed in the CS framework for the last decade, either promoting sparsity in an appropriate domain (total variation, wavelets, data-driven dictionary) or low-rank reconstruction as regularizing constraints [14-17]. CS-MRI has been shown to be more efficient in the high resolution setting [18], especially if a high Signalto-Noise Ratio (SNR) is preserved during data acquisition (i.e., input SNR) [19]. Practically, this constraint is fulfilled by collecting $k$ space measurements over a phased array coil and using these undersampled multi-channel data in the CS reconstruction. In this context, most reconstruction algorithms (e.g., CS-SENSE, sparse-SENSE) in the literature $[20,21]$ have been proposed for Cartesian data.

In this paper, our contribution is two-fold. First, it consists in filling the gap between CS and PI for non-Cartesian data by proposing a new self-calibrating method for automatically estimating the sensitivity maps. Second, we propose for the first time the application of POGM [22] to the CS-PI reconstruction problem and compare its performance with FB [23] and FISTA [24] on prospective 8, 12, 15fold under-sampled data collected at 7 Tesla for high resolution 2D $T_{2}^{*}$ brain imaging.

\section{PROBLEM STATEMENT}

In this work, we focus on the Sparse-SENSE (Sensitivity Encoding) formulation [20] of parallel imaging since it can be easily applied to non-Cartesian sampling schemes. 


\subsection{Combination of Parallel Imaging and Compressed Sensing}

Let $L$ be the number of coils used to acquire the Nuclear Magnetic Resonance (NMR) signal, $N$ be the number of pixels of the complex-valued image $\boldsymbol{x}$ to be reconstructed and $M$ the number of samples collected per channel during acquisition. We denote by $\boldsymbol{y}_{\ell} \in \mathbb{C}^{M}$ the complex-valued data recorded by the $\ell^{\text {th }}$ channel, $\boldsymbol{S}_{\ell} \in \mathbb{C}^{N \times N}$ the corresponding diagonal sensitivity matrix. Let $F$ be the NFFT and $\Omega \in\{1, \ldots, N\}$ the sampling pattern in the $k$-space, with $|\Omega|=M \ll N$. The CS-PI acquisition model thus reads: $\forall \ell=1: L, \boldsymbol{y}_{\ell}=F_{\Omega} \boldsymbol{S}_{\ell} \boldsymbol{x}+\boldsymbol{b}_{\ell}$ where $\boldsymbol{b}_{\ell}$ is additive zeromean Gaussian noise of variance $\sigma_{\ell}^{2}$, which can be characterized by a separate scan (without RF pulse) considering the same bandwidth as the prospective $\mathrm{CS}$ acquisition.

We also define the sparsifying transform $\boldsymbol{\Phi}$, such that the unknown image $\boldsymbol{x} \in \mathbb{C}^{N}$ is represented by a few non-zero coefficients $\boldsymbol{\alpha}=\boldsymbol{\Phi} \boldsymbol{x}$. Here, $\boldsymbol{\Phi}$ defines a orthogonal wavelet basis (Symmlet 8) but the presented work extends to redundant transforms such as curvelets or tight frames. In the Sparse-SENSE formulation, the reconstruction problem reads as the minimization of the $\ell_{1}$-analysisbased regularized criterion:

$$
\widehat{\boldsymbol{x}}=\underset{\boldsymbol{x} \in \mathbb{C}^{N}}{\arg \min }\left\{\frac{1}{2} \sum_{\ell=1}^{L} \sigma_{\ell}^{-2}\left\|F_{\Omega} \boldsymbol{S}_{\ell} \boldsymbol{x}-\boldsymbol{y}_{\ell}\right\|_{2}^{2}+\lambda\|\boldsymbol{\Phi} \boldsymbol{x}\|_{1}\right\},
$$

where $\lambda>0$ is the regularization parameter. This formulation requires the knowledge of the sensitivity maps $\left\{\boldsymbol{S}_{\ell}\right\}_{\ell}$. Several methods (e.g., ESPIRiT) have thus been proposed to extract them [25]. However, most of them were for Cartesian sampling schemes or consist in the non-Cartesian setting [26], in extracting the $k$-space center and applying gridding algorithms. In this work, we propose a self-calibrating method to extract the sensitivity maps for variable density non-Cartesian sampling schemes (see details hereafter). Next, instead of solving Eq. (1), we adopt the synthesis-based version which consists of minimizing it with respect to $\boldsymbol{\alpha}$ and then computing $\widehat{\boldsymbol{x}}=\boldsymbol{\Phi}^{*} \widehat{\boldsymbol{\alpha}}$ where $\boldsymbol{\Phi}^{*}$ is the adjoint (conjugate transpose) of $\boldsymbol{\Phi}$. Both formulations are equivalent as long as $\boldsymbol{\Phi}$ defines a basis, but the analysis-based formulation may provide improved solutions as $\boldsymbol{\Phi}$ is a tight frame. For minimizing this synthesis formulation, we then revisit the appropriate nonsmooth optimization algorithms in Sec. 3 and leave the comparison with primal-dual approaches [27-29], which are more dedicated to the analysis formulation, to future work. Also, we do not address the automatic tuning of $\lambda$, see for instance [30] to tackle this issue.

\subsection{Sensitivity maps extraction}

Since sensitivity maps information lies in the low-frequency domain, variable density sampling schemes like spirals, radials or sparkling [31] (see Fig. 1) intrinsically handle this information and thus are self-calibrating, namely they do not require the full acquisition of the $k$-space center. First, our sensitivity map estimation method relies on the extraction of the e.g. $\theta \%$ central surface of the collected measurements (here $\theta=10$ ) and the other measurements are set to zero. Second, low frequency $N \times N$ coil images are reconstructed applying the NFFT adjoint operator $F^{*}$ to the data completed by zero-filling: $\boldsymbol{z}_{\ell}=F_{\left[\Omega_{\mid \theta \%}^{\star}, \mathbf{0}\right]}^{\boldsymbol{y}_{\ell}}$. Third, the square root of the Sum of Squares (SOS) is computed: $\widehat{\boldsymbol{x}}^{\mathrm{LR}}=\sqrt{\sum_{\ell=1}^{L}\left\|\boldsymbol{z}_{\ell}\right\|^{2}}$ where LR stands for low resolution. Fourth, the sensitivity maps are given by the pixelwise ratio between image coils and the SOS: $\left[\boldsymbol{s}_{\ell}^{\mathrm{LR}}\right]_{i j}=\operatorname{diag}\left[\boldsymbol{S}_{\ell}\right]_{i j}=\left[\boldsymbol{z}_{\ell}\right]_{i j} /\left[\widehat{\boldsymbol{x}}^{\mathrm{LR}}\right]_{i j}, \forall \ell=1: L,(i, j=1:$ $N)$. Because of this SOS operation, our method less depends on the threshold $\theta$ compared to [26], who directly exploits the $\boldsymbol{z}_{\ell}$ images as sensitivity map information.

Noise attenuation in the image background is achieved by masking the estimated sensitivity maps. This binary mask is actually computed by thresholding $\widehat{\boldsymbol{x}}_{\mathrm{LR}}$, where the actual value of the threshold is given by a 2-cluster k-means algorithm. The binary mask is eventually defined as the largest connected component. (a) Sparkling

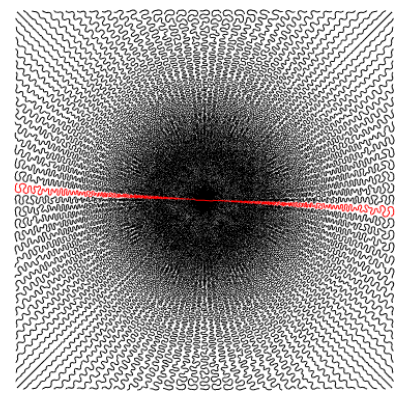

(b) Radial

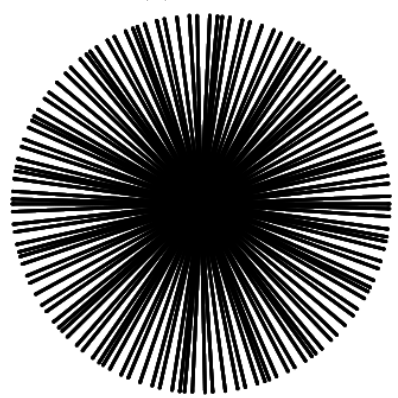

Fig. 1. Two non-Cartesian multi-shot under-sampling schemes.

\section{OPTIMIZATION ALGORITHM}

The CS-PI problem (1) is convex but nonsmooth since the $\ell_{1}$ penalty term is not differentiable at 0 . Also, minimizing Eq. (1) is computationally demanding in the high-resolution setting when $\Omega$ does not live on a Cartesian grid because the complexity of the NFFT is no longer in $O(N \log N)$ operations but instead in $O(N \log N+$ $\log (1 / \varepsilon) M)$ where $\varepsilon$ denotes the desired accuracy. Hence, fast optimization methods are required to reduce the number of iterations.

To solve problem (1), we compare proximal gradient methods [32] which basically include the FB splitting method, its acceleration [33] usually referred to as FISTA [24], and the most recent POGM [22] which is supposed to have a better worst-case convergence rate than FISTA. Note that our work proposes the first application of POGM to the CS-PI reconstruction problem.

Problem (1) reads as follows: $\widehat{\boldsymbol{\alpha}}=\arg \min _{\boldsymbol{\alpha} \in C^{N}}\{f(\boldsymbol{\alpha})+g(\boldsymbol{\alpha})\}$ where $f$ is a smooth $C^{1,1}$ (i.e., continuously differentiable) convex function with $\beta$-Lipschitz continuous gradient:

$$
\|\nabla f(x)-\nabla f(y)\| \leq \beta\|x-y\| \text { for every }(x, y) \in \mathbb{C}^{(N, N)}
$$

$g$ is a non-smooth continuous convex function and with an explicit proximity operator. In our case the functions are defined as follow:

$$
f(\boldsymbol{\alpha})=\frac{1}{2} \sum_{\ell=1}^{L} \sigma_{\ell}^{-2}\left\|F_{\Omega} \boldsymbol{S}_{\ell} \boldsymbol{\Phi}^{*} \boldsymbol{\alpha}-\boldsymbol{y}_{\ell}\right\|_{2}^{2}, \quad g(\boldsymbol{\alpha})=\lambda\|\boldsymbol{\alpha}\|_{1}
$$

The proximity operator of $g$ is the Soft-Thresholding operation. Importantly, because of the NFFT, the Lipschitz constant $\beta$ is computed using a power iteration method (eigenvalue decomposition).

The original FB algorithm is a generalization of gradient descent methods to non-differentiable functions. Hence it can be expressed as follows: $x_{k+1}=\operatorname{prox}_{\tau_{k} g}\left(x_{k}-\tau_{k} \nabla f\left(x_{k}\right)\right)$ where $\tau_{k}>0$ is a stepsize. FB accelerations can be summarized as follows:

$$
x_{k+1}=\operatorname{prox}_{\tau_{k} g}\left(y_{k}-\tau_{k} \nabla f\left(y_{k}\right)\right), \quad y_{k+1}=B_{k}\left(x_{k}, x_{k+1}\right)
$$

where operator $B_{k}$ denotes a linear combination - usually termed "momentum" rule - between the previous $x_{k}$ and current solutions 
$x_{k+1}$. The difference between FB accelerations lies in the coefficients involved in $B_{k}$.

For POGM, since the momentum rule is more technically involved, the pseudo-code is reported in Algorithm 1.

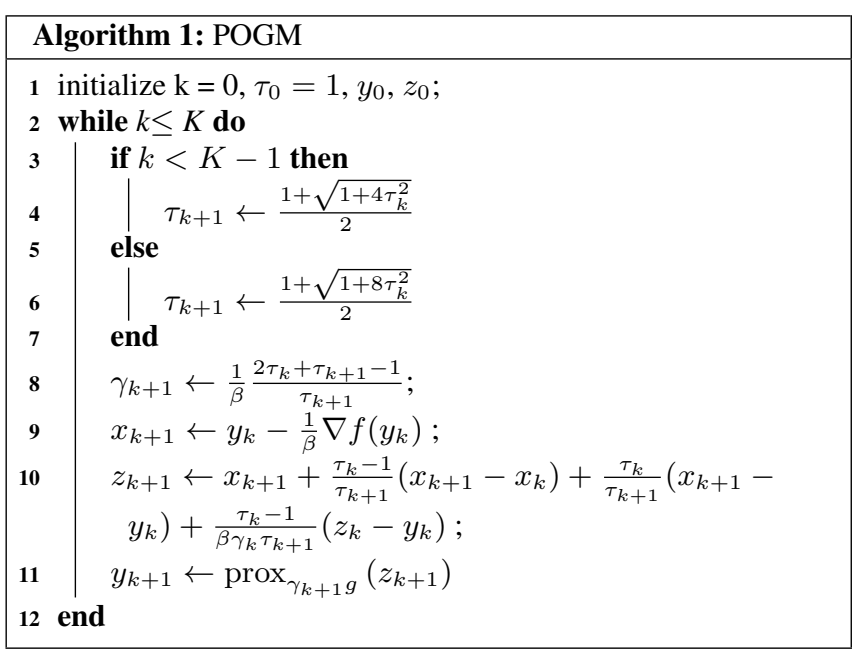

\section{RESULTS}

For validation purposes, we acquired 6 different brain anatomical MRI data at 7T (Magnetom Siemens scanner, Erlangen, Germany) using the 32-channel (Nova Medical Inc., Washington, MA, USA) coil (i.e., $L=32$ ). A modified $2 \mathrm{D}$ T $2 *$-weighted GRE sequence was implemented to perform prospective CS based on the multi-shot Sparkling trajectories [31]. The acquisition parameters were set as follows: $\mathrm{TR}=550 \mathrm{~ms}$, $\mathrm{TE}=30 \mathrm{~ms}$ and $\mathrm{FA}=25^{\circ}$ with inplane resolution of $400 \mu \mathrm{m}$ corresponding to an image matrix size of $N=512 \times 512$. Sparkling shots were generated all together using the algorithm proposed in [34] to draw samples according to a variable density with a polynomial decay of 2 (i.e., $h\left(k_{x}, k_{y}\right)=1 /|k|^{2}$ ). Specific adaptation to prospective MRI constraints [31] were implemented such as TE specification. The acceleration factor in time is given by the ratio between the number of shots and the number of shots in the reference (e.g. 512). For each acquisition the fully sampled reference of 512 Cartesian lines composed of 512 samples per lines was acquired too. Table 1 summarizes the acquisition parameters for the different datasets.

The regularization parameter $\lambda$ was tuned using a grid search procedure over a discrete set of values within $\left[10^{-7}, 10^{-4}\right]$. The selected value was the one giving the highest image quality with respect to the Structural Similarity (SSIM) metric [35]. All experiments were run on a machine with $128 \mathrm{~GB}$ of RAM and an 8core $(2.40 \mathrm{GHz})$ Intel Xeon E5-2630 v3 Processor.

Table 1. Ex-vivo baboon and in-vivo Human brain data collected using various slice thickness and acceleration factor $R$.

\begin{tabular}{|l|c|c|}
\hline Object & Slice thickness & $R$ \\
\hline 1. Baboon brain (ex-vivo) & $3 \mathrm{~mm}$ & $12(43$ shots $)$ \\
\hline 2. Baboon brain (ex-vivo) & $3 \mathrm{~mm}$ & $15(34$ shots $)$ \\
\hline 3. Baboon brain (ex-vivo) & $2 \mathrm{~mm}$ & $15(34$ shots $)$ \\
\hline 4. Baboon brain (ex-vivo) & $1 \mathrm{~mm}$ & $15(34$ shots) \\
\hline 5. Human brain (in-vivo) & $3 \mathrm{~mm}$ & $8(64$ shots) \\
\hline 6. Human brain (in-vivo) & $3 \mathrm{~mm}$ & $15(34$ shots $)$ \\
\hline
\end{tabular}
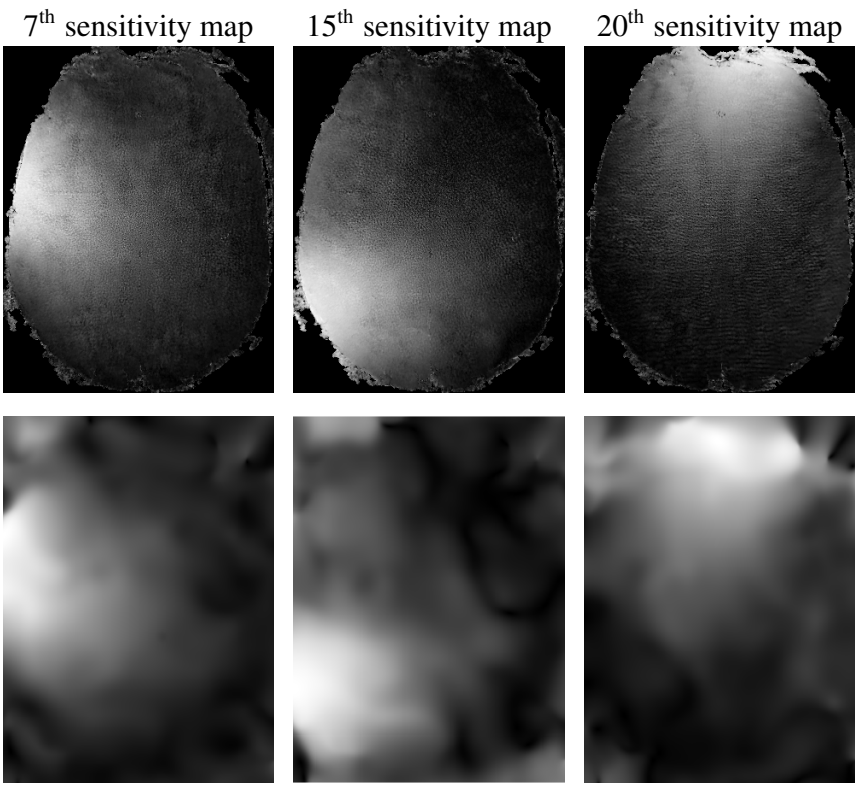

Fig. 2. Top: 3 out of 32 sensitivity maps extracted using our method. Bottom: Three representative sensitivity maps yielded by the ESPIRiT algorithm based on eigenvalue decomposition.

\subsection{Sensitivity maps extraction and MR image reconstruction}

Fig. 2 illustrates the result for the acquisition 5. for the sensitivity maps extraction on the same channels, three sensitivity maps extracted using the proposed method as well as those given by the $\ell_{1}$-ESPIRiT [25]. The latter is actually available in the BART toolbox [36]. The striking difference between the two approaches lies in the pattern of image-based representations of $\boldsymbol{S}_{\ell}$ matrices: because of the SVD decomposition involved in $\ell_{1}$-ESPIRiT, the corresponding sensitivity profiles are less structured and smoother than the maps yielded by our approach, which appear sharper and clearly indicate the FOV part illuminated by each channel. Moreover, our approach is much more efficient in terms of computing time since the average costs is about 1 min using a Matlab R2017a-based implementation as compared to $10 \mathrm{~min}$ for $\ell_{1}$-ESPIRiT on the same architecture and software.

Next for all the acquisition, MR images $(N=512 \times 512)$, either using our approach based on the extracted sensitivity maps and the POGM algorithm or using $\ell_{1}$-ESPIRiT. An example of the results for the acquisition 5. are depicted in Fig. 3. Although full FOV images reconstructed by POGM and $\ell_{1}$-ESPIRiT look very similar (Fig. 3(a)-(c)), the respective zooms (Fig. 3(d)-(f)) located in the posterior part of the human brain allow us to illustrate the smoothing effect induced by $\ell_{1}$-ESPIRiT: the fine details (i.e., dark stripes) we can observe in the white matter on the Cartesian reference are lost in the $\ell_{1}$-ESPIRiT image whereas they are quite well preserved using our self-calibrating solution. In addition, the convergence speed of our reconstruction was of $35 \mathrm{~min}$ whereas $\ell_{1}$-ESPIRiT took about 1 hour to provide the reconstructed image. For the six acquisitions that have been tested, we report in Table 2 the SSIM values for both approaches.

\subsection{Convergence speed}

To assess the convergence speed of the FB, FISTA and POGM algorithms, we performed a numerical experiment using the same setup, i.e. identical $\lambda$ values, same initializations $\boldsymbol{x}^{(0)}$ and same $\left\{\widehat{\boldsymbol{S}}_{\ell}\right\}_{\ell=1}^{L}$ estimates $(L=32)$. Fig. 4 shows the convergence speed for FISTA, 
(a)

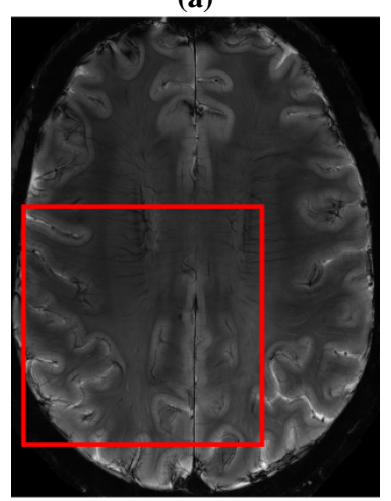

(d)

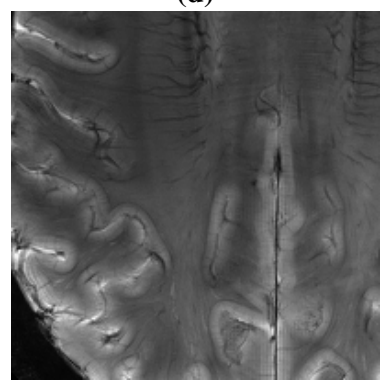

(b)

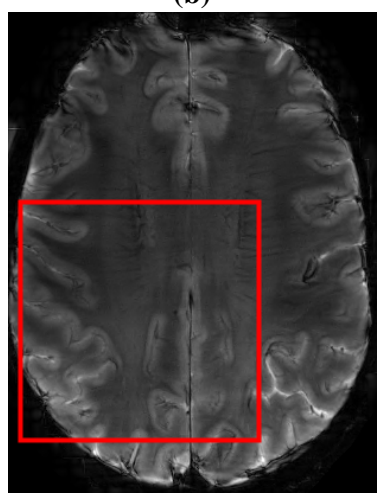

(e)

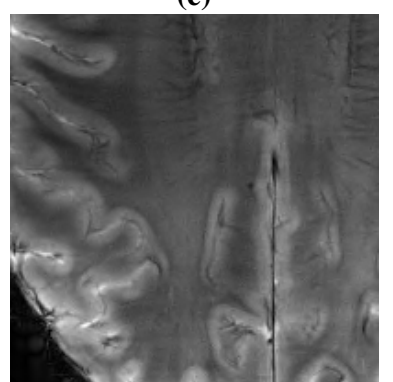

(c)

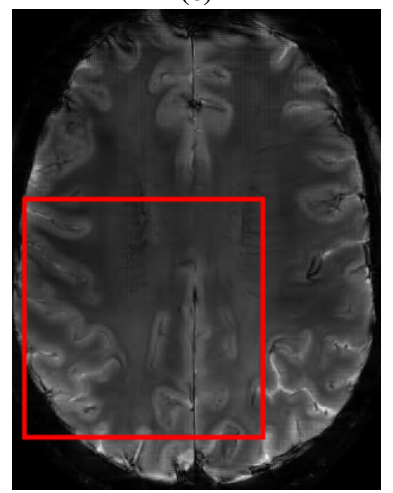

(f)

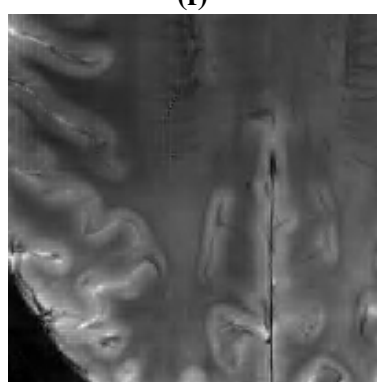

Fig. 3. (a) Cartesian reference, (b) Self-calibrating POGM-based and (c) $\ell_{1}$-ESPIRiT reconstructions from 8 -fold accelerated prospective CS based on Sparkling trajectories. (d)-(f) respective zooms in the red square.

Table 2. SSIM values within the $(0,1)$ range for the proposed and ESPIRiT methods computed over the 6 datasets. The larger the SSIM the better the image quality. Bold font indicates the best score.

\begin{tabular}{|c|c|c|}
\hline Dataset & $\ell_{1}$-ESPIRiT & Proposed approach \\
\hline 1. & 0.83 & $\mathbf{0 . 8 6}$ \\
\hline 2. & 0.77 & $\mathbf{0 . 7 8}$ \\
\hline 3. & 0.84 & $\mathbf{0 . 8 5}$ \\
\hline 4. & 0.81 & $\mathbf{0 . 8 3}$ \\
\hline $\mathbf{5 .}$ & 0.75 & 0.75 \\
\hline 6. & 0.75 & $\mathbf{0 . 7 6}$ \\
\hline
\end{tabular}

POGM and the original FB implementation. To track numerical convergence, we computed at each iteration $k$ the following normalized cost function $\epsilon$ :

$$
\epsilon(k)=10 \log _{10}\left(\frac{\left\|F\left(x_{k}\right)-F\left(x^{*}\right)\right\|_{2}^{2}}{\left\|F\left(x^{*}\right)\right\|_{2}^{2}}\right)
$$

where $F(\cdot)=f(\cdot)+g(\cdot)$ is the global cost function to minimize (cf. Eq. (3)) and $x^{*}$ is the converged solution obtained by running $10^{3}$ iterations. As seen on the curves in Fig. 4, the accelerations of the initial FB algorithm show some well known behavior referred to as 'Nesterov ripples' even though both FISTA and POGM decrease faster than FB. Interestingly, POGM decreases a little bit faster than FISTA especially during the first tens of iterations. Also, the length of rippling cycles in POGM is smaller than in FISTA even though they seem more frequent in POGM.

\section{CONCLUSION}

In this work, we have proposed a new self-calibrating method to estimate the sensitivity maps from non-Cartesian variable density under-

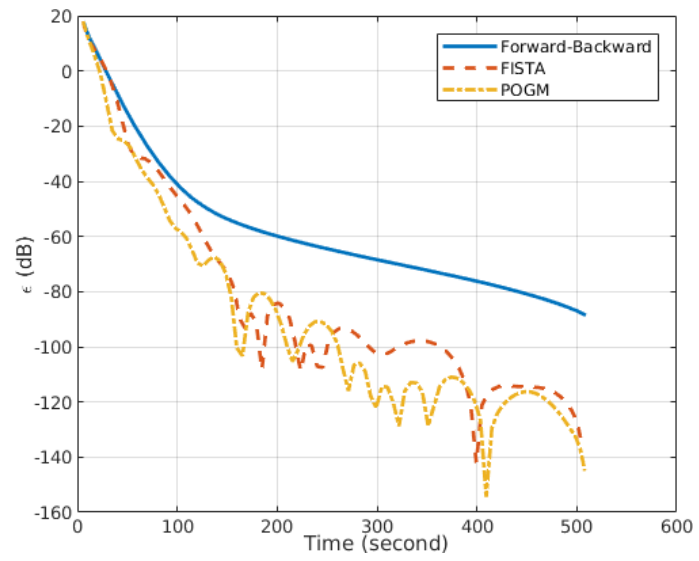

Fig. 4. Comparison of convergence speed for the different optimization algorithms: FB, FISTA and POGM which relie on the same estimated sensitivity maps.

sampling schemes. Compared to the state-of-the-art, our approach is much more efficient and the sensitivity profiles are easier to interpret. Based on these estimates, we have also compared three proximal gradient methods that provide the same reconstructed MR images but in different computing times: POGM converges slightly faster than FISTA whereas both outperform the original forward-backward algorithm. Noticeably, we applied for the first time the POGM algorithm to the CS reconstruction problem in a non-Cartesian and parallel reception setting. Still regarding reconstruction, we demonstrated on several anatomical $\mathrm{T}_{2}^{*}$ data collected at 7 Tesla that our global self-calibrating method is both more accurate and more efficient (by about a factor of 2 ) than the competing alternative $\ell_{1}$-ESPIRiT. 


\section{REFERENCES}

[1] K. P Pruessmann, M. Weiger, M. B Scheidegger, P. Boesiger, et al., "SENSE: sensitivity encoding for fast MRI," Magn. Reson. Med., vol. 42, no. 5, pp. 952-962, 1999.

[2] MA Griswold, PM Jakob, RM Heidemann, M. Nittka, V. Jellus, J. Wang, B. Kiefer, and A. Haase, "Generalized autocalibrating partially parallel acquisitions (GRAPPA)," Magn. Reson. Med., vol. 47, no. 6, pp. 1202-1210, 2002.

[3] I. Y. Chun, B. Adcock, and T. M. Talavage, "Efficient compressed sensing SENSE pMRI reconstruction with joint sparsity promotion," IEEE Trans. Med. Imag., vol. 35, no. 1, pp. 354-368, Jan 2016.

[4] D. L Donoho, “Compressed sensing," IEEE Trans. Inf. Theory, vol. 52, no. 4, pp. 1289-1306, 2006.

[5] E. J Candès, J. Romberg, and T. Tao, "Robust uncertainty principles: Exact signal reconstruction from highly incomplete frequency information," IEEE Trans. Inf. Theory, vol. 52, no. 2, pp. 489-509, 2006.

[6] M. Lustig, D. Donoho, and JM Pauly, "Sparse MRI: The application of compressed sensing for rapid MR imaging," Magn. Reson. Med., vol. 58, no. 6, pp. 1182-1195, 2007.

[7] G. Puy, P. Vandergheynst, and Y. Wiaux, "On variable density compressive sampling," IEEE Trans. Signal Proc. Let., vol. 18, no. 10, pp. 595-598, 2011.

[8] N. Chauffert, P. Ciuciu, and P. Weiss, "Variable density compressed sensing in MRI. Theoretical vs heuristic sampling strategies," in 10th Proc. IEEE ISBI, San Francisco, CA, Apr. 2013, pp. 298-301.

[9] N. Chauffert, P. Ciuciu, J. Kahn, and P. Weiss, "Variable density sampling with continuous trajectories. Application to MRI," SIAM J. Imag. Sci., vol. 7, no. 4, pp. 1962-1992, Nov. 2014.

[10] GH Glover and JM Pauly, "Projection reconstruction techniques for reduction of motion effects in MRI," Magn. Reson. Med., vol. 28, no. 2, pp. 275-289, 1992.

[11] CH Meyer, B.S Hu, D.G Nishimura, and A. Macovski, "Fast spiral coronary artery imaging," Magn. Reson. Med., vol. 28, no. 2, pp. 202213, 1992.

[12] J. Keiner, S. Kunis, and D. Potts, "Using NFFT 3-a software library for various nonequispaced fast Fourier transforms," ACM Trans. on Math. Soft., vol. 36, no. 4, pp. 19, 2009.

[13] J. A Fessler and B. P Sutton, "Nonuniform fast Fourier transforms using min-max interpolation," IEEE Trans. Signal Proc., vol. 51, no. 2, pp. 560-574, 2003.

[14] M. Guerquin-Kern, M. Haberlin, K. Pruessmann, and M. Unser, "A fast wavelet-based reconstruction method for magnetic resonance imaging," IEEE Trans. Med. Imag., vol. 30, no. 9, pp. 1649-1660, 2011.

[15] L. Chaari, J.-C. Pesquet, A. Benazza-Benyahia, and P. Ciuciu, "A wavelet-based regularized reconstruction algorithm for SENSE parallel MRI with applications to neuroimaging," MedIA, vol. 15, no. 2, pp. 185-201, 2011.

[16] B. Zhao, J. P Haldar, A. G Christodoulou, and Z.-P. Liang, "Image reconstruction from highly undersampled $(k, t)$-space data with joint partial separability and sparsity constraints," IEEE Trans. Med. Imag., vol. 31, no. 9, pp. 1809-1820, 2012.

[17] J. P Haldar, "Low-rank modeling of local $k$-space neighborhoods (LORAKS) for constrained MRI," IEEE Trans. Med. Imag., vol. 33, no. 3, pp. 668-681, 2014.

[18] B. Adcock, AC Hansen, C. Poon, and B. Roman, "Breaking the coherence barrier: A new theory for compressed sensing," vol. 5, 2017.

[19] C. Lazarus, P. Weiss, A. Vignaud, and P. Ciuciu, "An empirical study of the maximum degree of acceleration in Compressed Sensing MRI," submitted to Magn. Reson. Med., CEA/NeuroSpin \& INRIA Saclay Parietal, Saclay, France, June 2017.

[20] D. Liang, B. Liu, J. Wang, and L. Ying, "Accelerating SENSE using compressed sensing," Magn. Reson. Med., vol. 62, no. 6, pp. 1574 1584, 2009.
[21] F. Huang, Y. Chen, W. Yin, W. Lin, X. Ye, W. Guo, and A. Reykowski, "A rapid and robust numerical algorithm for sensitivity encoding with sparsity constraints: Self-feeding sparse SENSE," Magn. Reson. Med., vol. 64, no. 4, pp. 1078-1088, 2010.

[22] A. B Taylor, J. M Hendrickx, and F. Glineur, "Exact worst-case convergence rates of the proximal gradient method for composite convex minimization," preprint arXiv:1705.04398, 2017.

[23] Patrick L Combettes and Valérie R Wajs, "Signal recovery by proximal forward-backward splitting," Multiscale Modeling \& Simulation, vol. 4, no. 4, pp. 1168-1200, 2005.

[24] A. Beck and M. Teboulle, "Fast gradient-based algorithms for constrained total variation image denoising and deblurring problems," IEEE Trans. Image Process., vol. 18, no. 11, pp. 2419-2434, 2009.

[25] M. Uecker, P. Lai, M. J Murphy, P. Virtue, M. Elad, J. M Pauly, S. S Vasanawala, and M. Lustig, "ESPIRiTAn Eigenvalue Approach to Autocalibrating Parallel MRI," Magn. Reson. Med., vol. 71, pp. 990-1001, 2014.

[26] EN Yeh, M. Stuber, CA McKenzie, RM Botnar, T. Leiner, MA Ohliger, AK Grant, J D Willig-Onwuachi, and D K Sodickson, "Inherently selfcalibrating non-Cartesian parallel imaging," Magn. Reson. Med., vol. 54, no. 1, pp. 1-8, 2005.

[27] A. Chambolle, "On a class of first-order algorithms for convex problems with applications to imaging," Isis, pp. 1-49, 2010.

[28] Bng Công Vũ, "A splitting algorithm for dual monotone inclusions involving cocoercive operators," Advances in Computational Mathematics, vol. 38, no. 3, pp. 667-681, Apr 2013.

[29] L. Condat, "A primal-dual splitting method for convex optimization involving lipschitzian, proximable and linear composite terms," Journal of Optimization Theory and Applications, vol. 158, no. 2, pp. 460-479, Aug 2013.

[30] S. Ramani, Z. Liu, J. Rosen, JF Nielsen, and JA Fessler, "Regularization parameter selection for nonlinear iterative image restoration and mri reconstruction using GCV and SURE-based methods," IEEE Trans. Image Proc., vol. 21, no. 8, pp. 3659-3672, 2012.

[31] C. Lazarus, P. Weiss, N. Chauffert, F. Mauconduit, M. Bottlaender, A. Vignaud, and P. Ciuciu, "SPARKLING: Novel Non-Cartesian Sampling Schemes for Accelerated 2D Anatomical Imaging at 7T Using Compressed Sensing," in 25th annual meeting of the ISMRM, Honolulu, United States, 2017

[32] PL Combettes and J-C Pesquet, "Proximal splitting methods in signal processing," in Fixed-point algorithms for inverse problems in science and engineering, pp. 185-212. Springer, 2011.

[33] Y. Nesterov et al., "Gradient methods for minimizing composite objective function," 2007.

[34] C. Boyer, N. Chauffert, P. Ciuciu, J. Kahn, and P. Weiss, "On the generation of sampling schemes for magnetic resonance imaging," SIAM J. Imag. Sci., vol. 9, no. 4, pp. 2039-2072, 2016.

[35] Z. Wang, AC Bovik, H R Sheikh, and EP Simoncelli, "Image quality assessment: from error visibility to structural similarity," IEEE Transactions on Image Processing, vol. 13, no. 4, pp. 600-612, 2004.

[36] M. Uecker, F. Ong, J. I Tamir, D. Bahri, P. Virtue, J. Y Cheng, T. Zhang, and M. Lustig, "Berkeley advanced reconstruction toolbox," in Proc. Intl. Soc. Mag. Reson. Med, 2015, vol. 23, p. 2486. 